\title{
Variables predictoras de riesgo de trastorno del comportamiento alimentario en mujeres
}

\author{
Predictors of risk for eating disorders in women
}

\author{
Karina Franco \\ Felipe de Jesús Díaz \\ Antonio López-Espinoza \\ Centro Universitario del Sur, Universidad de Guadalajara, Jalisco, México. \\ María del Consuelo Escoto \\ Esteban Jaime Camacho \\ Centro Universitario UAEM Ecatepec, Universidad Autónoma de Estado de México \\ Unidad Académica Profesional de Netzahualcóyotl, Universidad Autónoma del Estado de México
}

(Rec: 11 de julio de 2011/Acept: 16 de abril de 2013)

\begin{abstract}
Resumen
El propósito de esta investigación fue analizar el papel de la composición corporal, la insatisfacción corporal y el modelo de delgadez sobre el riesgo de desarrollar trastornos del comportamiento alimentario (TCA). Participaron 289 estudiantes universitarias, quienes contestaron el Cuestionario de Actitudes Alimentarias, el Cuestionario de Bulimia de Edimburgo y el Cuestionario de Influencias del Modelo Estético Corporal. La composición corporal se analizó por medio de bioimpedancia eléctrica. Se encontró que 9.69\% de las mujeres presentaron riesgo de TCA, siendo mayor el porcentaje entre las mujeres que tenían peso normal y cantidades excesivas de grasa corporal. La insatisfacción corporal predijo el riesgo de anorexia nerviosa, y la interacción entre insatisfacción corporal, influencia de la publicidad e índice de masa corporal predijo el riesgo de bulimia nerviosa. Se concluye que la insatisfacción corporal juega un papel relevante en la predicción de riesgo de TCA.

Palabras clave: composición corporal, modelo de delgadez, insatisfacción corporal, riesgo de trastorno del comportamiento alimentario y jóvenes.
\end{abstract}

\begin{abstract}
The purpose of this research was to examine the role of body composition, body dissatisfaction, and thinness model on the risk of developing eating disorders (ED). The sample comprised 289 female students who answered the Eating Attitudes Test, the Bulimic Investigatory Test, Edinburgh, and the Questionnaire on Influences on Body Shape Model. The body composition was assessed by electrical impedance. The results showed that the risk for having an ED was found in $9.69 \%$ of women, and the percentage was high among normal weight women, especially women who had excessive body fat. The body dissatisfaction predicted the risk for anorexia nervosa, and the interaction between body dissatisfaction, influence of advertisement and body mass index predicted the risk for bulimia nervosa. These findings suggest that the role of body dissatisfaction was relevant to predict the risk for ED.

Key words: body composition, thinness model, body dissatisfaction, eating disorders risk, young.
\end{abstract}

Nota de los autores: Los autores agradecen el financiamiento PROMEP/103.5/09/3912 otorgado a la Dra. Karina Franco Paredes para la realización de esta investigación. También agradecen a Gabriela Zepeda Plazola; Sandra Yadira Isais Cabadas, Rosa Elena Ramírez Ruelas, Iván Alejandro Muñiz González y Julio César Romero Parra su apoyo para la evaluación de las participantes en esta investigación.

Correspondencia: Karina Franco Paredes, Centro de Investigaciones en Comportamiento Alimentario y Nutrición, CUSur-Universidad de Guadalajara. Av. Prolongación Colón s/n, C.P. 49000, Ciudad Guzmán, Jalisco, México. Correo electrónico: karina.franco@cusur.udg.mx 


\section{Introducción}

El estudio de las conductas alimentarias de riesgo para el desarrollo de trastornos del comportamiento alimentario (TCA) en población mexicana ha mostrado que cantidades importantes de jóvenes modifican su alimentación o su conducta con el propósito de controlar el peso corporal. Los resultados de dos encuestas son relevantes debido a que evaluaron conductas relacionadas con la alimentación y el peso corporal incluyendo muestras de jóvenes de todos los estados de la República Mexicana. En la Encuesta Nacional de la Juventud 2005 (Pérez, Valdez y Serrano, 2006) se encontró que $23.6 \%$ de los jóvenes entre 12 y 29 años de edad estaban inconformes con su peso, hecho que se relacionó positivamente con la edad. En tanto que en la Encuesta Nacional de Salud y Nutrición (ENSANUT; Olaiz-Fernández et al., 2006) se encontró que la prevalencia de conductas alimentarias de riesgo fue de $0.7 \%$. Posteriormente, se llevó a cabo un análisis más detallado de los datos obtenidos en la ENSANUT con la finalidad de calcular la prevalencia de riesgo de TCA. Se estudió la información de 25.056 adolescentes entre 10 y 19 años de edad y se encontró que $0.8 \%$ de los adolescentes presentaron riesgo de TCA (Barriguete-Meléndez et al., 2009). Este resultado es similar al obtenido en un estudio en el que se evaluó la prevalencia de riesgo de TCA en mujeres en dos momentos, 1995 y 2002, (Mancilla-Díaz et al., 2007).

Asimismo, estudios realizados en diferentes estados de la República Mexicana han encontrado que jóvenes de la Ciudad de México y su zona metropolitana (Álvarez et al., 2003; Mancilla-Díaz et al., 2009; Unikel-Santoncini, Bojórquez-Chapela, Villatoro-Velázquez, Fleiz-Bautista y Medina-Mora, 2006; Unikel, Saucedo-Molina, Villatoro y Fleiz, 2002; Unikel et al., 2000; Vázquez et al., 2005) y de los estados de Aguascalientes (Martínez, Álvarez, Góngora, Franco y Mancilla, 2009); Guanajuato (Chávez, Macías, Gutiérrez, Martínez y Ojeda, 2004); Hidalgo (Saucedo-Molina, Escamilla-Talón, Portillo-Noriega, Peña-Irecta y CalderónRamos, 2008; Saucedo-Molina y Unikel, 2010), Jalisco (Franco, Martínez et al., 2010; Nuño-Gutiérrez, Celis-De la Rosa y Unikel-Santoncini, 2009), Michoacán (Bojórquez y Unikel, 2004), Nuevo León (Alvarez et al., 2003) y Querétaro (Aguilera y Milián, 2006) presentan conductas alimentarias que suponen un riesgo para el desarrollo de TCA (e.g. dieta, atracón, consumo de producto para adelgazar, ejercicio para quemar calorías, vómito, uso de laxantes). Los resultados de dos estudios multicéntricos que incluyeron muestras de jóvenes de diferentes estados brindan una perspectiva general sobre la cantidad de jóvenes en riesgo de desarrollar alguna psicopatología alimentaria. Por ejemplo, Franco et al., 2007 evaluaron a jóvenes de cinco estados (Distrito Federal, Estado de México, Guanajuato, Puebla y Nuevo León) y encontraron que $14.34 \%$ de las mujeres presentaban riesgo de TCA. En tanto que Unikel-Santoncini et al. (2010) evaluaron mujeres de 17 estados (Aguascalientes, Colima, Chiapas, Chihuahua, Distrito Federal, Durango, Estado de México, Guanajuato, Guerrero, Hidalgo, Jalisco, Michoacán, Nayarit, Nuevo León, Tamaulipas, Oaxaca y Veracruz) y encontraron que la prevalencia total de riesgo moderado fue de $14.2 \%$ y de riesgo alto fue de $6.8 \%$.

Por otro lado, la influencia del modelo de delgadez y la insatisfacción corporal son dos variables relacionadas con el riesgo de desarrollar TCA. La promoción del modelo de delgadez como el ideal de belleza asociado a éxito, control y perfección ha generado que una cantidad importante de jóvenes, especialmente mujeres, se sientan insatisfechas con su imagen corporal (Toro, 2004), la cual es una característica principal de los TCA. Actualmente, la insatisfacción corporal se presenta de manera generalizada en las jóvenes, entre 14 y $25 \%$ de las mujeres informan estar insatisfechas con las dimensiones de su cuerpo (Álvarez, Nieto, Mancilla, Vázquez y Ocampo, 2007; Franco, Martínez et al., 2010; Vázquez, López, Álvarez, Franco y Mancilla, 2004; Vázquez et al., 2005). Asimismo, Los estudios han señalado que la insatisfacción corporal predice la dieta restrictiva (SaucedoMolina y Gómez, 2004), la presencia de TCA (Vázquez et al., 2005) o el riesgo de TCA (Álvarez, Franco, Mancilla, Álvarez y López, 1998); y se ha observado que las mujeres que realizan dietas restrictivas están más insatisfechas con su cuerpo y tienen más grasa corporal que quienes no realizan este tipo de dietas (Gruber, Pope, Lalonde y Hudson, 2001).

La composición corporal de las personas es otra variable que está relacionada con el riesgo de TCA. Se ha documentado que índices elevados de adiposidad resultan en un incremento tanto en la influencia del modelo de delgadez como en la insatisfacción corporal y, consecuentemente, aumenta el riesgo de TCA (Cattarin y Thompson, 1994). También se sabe que las personas con sobrepeso u obesidad presentan conductas alimentarias de riesgo en mayor medida que las personas con peso normal. No obstante, al correlacionar el Índice de Masa Corporal (IMC) con la presencia de indicadores clínicos de TCA (e.g. dieta restrictiva, insatisfacción corporal, atracón) los resultados han sido inconsistentes debido a que en algunos estudios se encuentran asociaciones estadísticamente significativas y en otros no (Saucedo-Molina y Gómez, 2004; SaucedoMolina et al., 2008; Saucedo-Molina y Unikel, 2010; Unikel et al., 2002). Respecto al papel que juega el IMC en los 
TCA, Stice (2002) sugiere que debe ser considerado un factor de riesgo para la influencia del modelo de delgadez, la insatisfacción corporal y la dieta, pero no un factor de riesgo directo de los TCA.

De manera particular, los resultados correspondientes al Estado de Jalisco, México, provenientes de la ENSANUT (Olaiz-Fernández et al., 2006), de un estudio multicéntrico (Unikel-Santoncini et al., 2010) y de investigaciones locales (Franco, Martínez et al., 2010; Nuño-Gutiérrez et al., 2009) han mostrado que una cantidad importante de mujeres presenta riesgo de TCA. Aún cuando se han utilizado instrumentos diferentes, las investigaciones coinciden en que alrededor de $10 \%$ de las mujeres se encuentran en esta condición (Franco, Martínez et al., 2010; Nuño-Gutiérrez et al., 2009). Por tal motivo, resulta relevante investigar qué variables están relacionadas con la presencia del riesgo de TCA. Por ello, el propósito de esta investigación fue analizar el papel de la composición corporal, la insatisfacción corporal y el modelo delgadez sobre el riesgo de desarrollar TCA en mujeres.

\section{Método}

\section{Participantes}

La muestra estuvo conformada por 289 mujeres estudiantes en una universidad pública del Sur del Estado de Jalisco, México, quienes aceptaron participar voluntariamente en el estudio. La edad promedio de las mujeres fue de 20.51 años ( $D E=1.24)$. El tamaño de la muestra fue determinado por conveniencia y el tipo de muestro fue no probabilístico de tipo intencional.

\section{Instrumentos}

Cuestionario de Actitudes Alimentarias (EAT-40, por sus siglas en inglés; Garner y Garfinkel, 1979) fue elaborado para medir síntomas y conductas características de anorexia nerviosa (AN), incluye 40 reactivos con seis opciones de respuesta en escala tipo Likert. En mujeres mexicanas el EAT-40 mostró una consistencia interna adecuada ( $\alpha=$ $.90)$, se estableció el punto de corte de 28 como indicador de riesgo de TCA (Álvarez, Vázquez, Mancilla y GómezPeresmitre, 2002) y se identificó una estructura de cinco factores: restricción alimentaria, motivación para adelgazar, bulimia, preocupación por la comida y presión social percibida (Álvarez et al., 2004).

Cuestionario de Bulimia de Edimburgo (BITE, por sus siglas en inglés; Henderson y Freeman, 1987) elaborado para medir síntomas y características de bulimia nerviosa
(BN) así como su severidad. El BITE está conformado por 30 reactivos con dos opciones de respuesta para evaluar síntomas de $\mathrm{BN}$ y tres reactivos en un formato de cinco a siete opciones de respuesta para evaluar la severidad del trastorno. La consistencia interna de este cuestionario fue apropiada tanto en mujeres mexicanas con TCA $(\alpha=.83)$ como en estudiantes universitarias $(\alpha=.85-.90)$ y se estableció el punto de corte de 21 como indicador de riesgo de TCA (Franco, González et al., 2010).

Cuestionario de Imagen Corporal (BSQ, por sus siglas en inglés; Cooper, Taylor, Cooper y Fairburn, 1987). Evalúa la percepción que una persona tiene de su cuerpo e identifica si presenta insatisfacción corporal. El BSQ está conformado por 34 reactivos con seis opciones de respuesta en escala tipo Likert. Las investigaciones en México han mostrado que la consistencia interna del instrumento en mujeres es excelente $(\alpha=.98)$ y se estableció un punto de corte de 110 , el cual indica presencia de insatisfacción corporal (Vázquez et al., 2004).

Cuestionario de Influencias del Modelo Estético Corporal (CIMEC; Toro, Salamero y Martínez, 1994). Evalúa la influencia de los agentes y situaciones sociales en las que se promueve el modelo estético de delgadez. EL CIMEC está conformado por 40 reactivos con tres opciones de respuesta. Vázquez, Álvarez y Mancilla (2000) analizaron las propiedades psicométricas del CIMEC en mujeres mexicanas; encontraron una consistencia interna adecuada $(\alpha=.94)$ y derivaron una estructura de cuatro factores: Influencia de la publicidad, Malestar con la imagen corporal, Influencia de los modelos estéticos corporales e Influencia de las relaciones sociales.

Tallímetro Tanita HR200, escala métrica que permite obtener la estatura o la longitud de un individuo, con una precisión de $1 \mathrm{~mm}$.

Analizador de Bioimpedancia InBody 230 con seis frecuencias de impedancia y tres de reactancia. Proporciona los indicadores antropométricos siguientes: peso, masa muscular, masa grasa, porcentaje de grasa e índice de masa corporal.

\section{Procedimiento}

La aplicación de los cuestionarios se llevó a cabo de manera grupal y en las aulas de la institución educativa. En primer lugar se les explicó el propósito de la investigación a las mujeres y quienes aceptaron participar, previo consentimiento informado por escrito, contestaron los instrumentos en aproximadamente 30 minutos. Uno de los investigadores permaneció en el aula durante la aplicación para responder cualquier pregunta y para verificar que los cuestionarios fueran contestados completamente. Personal capacitado registró 
las medidas antropométricas en un consultorio el mismo día de la aplicación de los cuestionarios. Para el registro de las medidas las participantes vestían ropa ligera y sin zapatos.

\section{Análisis de datos}

El análisis de datos se llevó a cabo en el Statistical Package for Social Sciences (SPSS). Los resultados del IMC y del porcentaje de grasa fueron analizados considerando la clasificación propuesta por la Organización Mundial de la Salud (OMS, 2003). Tomando en cuenta los puntos de corte del EAT y del BITE se calculó el porcentaje de personas que presentaron riesgo de TCA. Se llevó a cabo una prueba $t$ de Student para analizar si existían diferencias entre las mujeres con riesgo de TCA y las mujeres sin riesgo en cuanto al total del BSQ, CIMEC y sus factores, IMC y porcentaje de grasa. Finalmente, se llevó a cabo un análisis de regresión lineal múltiple para identificar las variables que predicen el riesgo de $\mathrm{AN}$ (EAT-40) o $\mathrm{BN}$ (BITE).

\section{Resultados}

El análisis de las medidas antropométricas de las participantes reveló que el promedio del IMC fue de $22.90(D E=$ 4.13), en tanto que el promedio del porcentaje de grasa fue $27.99(D E=8.19)$. Al clasificar a las participantes de acuerdo al IMC se encontró que $9.39 \%$ presentaron bajo peso, $66.06 \%$ peso normal, $18.41 \%$ sobrepeso y $6.14 \%$ obesidad. En la Tabla 1 se presenta el análisis del porcentaje de grasa corporal de acuerdo a la clasificación del peso corporal. Como se observa, $19.23 \%$ de las mujeres con bajo peso presentaron porcentaje normal de grasa corporal. En el caso de las mujeres con peso normal, $12.57 \%$ presentaron porcentaje elevado o excesivo de grasa. En las mujeres con sobrepeso, $11.76 \%$ y $74.51 \%$ presentaron moderados o excesivos porcentajes de grasa, respectivamente. Finalmente, $100 \%$ de las mujeres con obesidad presentaron porcentajes elevados o excesivos de grasa.

Tabla 1. Distribución porcentual del porcentaje de grasa de acuerdo a la clasificación del peso corporal.

\begin{tabular}{lcccc}
\hline \multirow{2}{*}{$\begin{array}{l}\text { Clasificación del peso } \\
\text { corporal }\end{array}$} & \multicolumn{5}{c}{ Porcentaje de grasa } \\
\cline { 2 - 5 } & Bajo & Normal & Elevado & Excesivo \\
\hline Bajo $\mathrm{n}=26$ & 80.77 & 19.23 & 0 & 0 \\
Normal $\mathrm{n}=195$ & 12.57 & 62.30 & 12.57 & 12.57 \\
Sobrepeso $\mathrm{n}=51$ & 0 & 13.73 & 11.76 & 74.51 \\
Obesidad $\mathrm{n}=17$ & 0 & 0 & 5.88 & 94.12 \\
\hline
\end{tabular}

Considerando la puntuación total del EAT-40, del BITE y del BSQ se encontró que $4.49 \%$ de las mujeres rebasaron el punto de corte del EAT, 3.80\% el del BITE y $1.40 \%$ el de ambos instrumentos; lo cual resultó en un total de $9.69 \%$ de mujeres con riesgo de TCA. Asimismo, 12.10\% de las mujeres presentaron insatisfacción corporal, de las cuales $51.4 \%$ presentaron riesgo de TCA y $48.6 \%$ no presentaron riesgo de TCA. Para las mujeres con riesgo se calculó la distribución porcentual de acuerdo al IMC y el porcentaje de grasa (véase Tabla 2). Como se observa, la mitad de los casos con riesgo de TCA se presentaron entre quienes tenían peso normal y en mayor porcentaje entre quienes presentaron niveles excesivos de grasa. El riesgo de TCA también estuvo presente en un porcentaje importante de mujeres con sobrepeso (32.14\%), específicamente entre quienes presentaron porcentaje excesivo de grasa $(22.43 \%)$. En general, los mayores porcentajes de riesgo de TCA se presentaron entre quienes tenían porcentaje elevado o excesivo de grasa.

Tabla 2. Distribución porcentual del riesgo de trastornos del comportamiento alimentario de acuerdo al índice de masa corporal y el porcentaje de grasa.

\begin{tabular}{lccccc}
\hline Clasificación del & \multicolumn{5}{c}{ Porcentaje de grasa } \\
\cline { 2 - 5 } peso corporal & Bajo & Normal & Elevado & Excesivo & Total \\
\hline Bajo & 3.57 & 3.57 & 0 & 0 & 7.14 \\
Normal & 3.57 & 14.29 & 10.71 & 22.43 & 50 \\
Sobrepeso & 0 & 7.14 & 3.57 & 22.43 & 32.14 \\
Obesidad & 0 & 0 & 0 & 10.71 & 10.71 \\
Total & 7.14 & 25 & 14.28 & 53.57 & 100 \\
\hline
\end{tabular}

En la Tabla 3 se presenta la comparación de las puntuaciones promedio para el total del BSQ, del CIMEC y sus respectivos factores, y de la composición corporal (IMC y porcentaje de grasa) entre las mujeres con riesgo de TCA y sin riesgo. Como se observa las mujeres con riesgo presentaron puntuaciones promedio significativamente mayores que las mujeres sin riesgo en el total del BSQ, CIMEC y sus factores, así como en el porcentaje de grasa; en tanto que la diferencia fue marginalmente significativa en el IMC.

Se llevó a cabo un análisis de regresión lineal múltiple para evaluar si las variables insatisfacción corporal (BSQ), influencia de la publicidad, influencia de los modelos sociales e influencia de las relaciones sociales (factores del CIMEC), IMC y porcentaje de grasa mostraban capacidad para explicar el riesgo de AN (EAT-40) y BN (BITE). Como se observa en la Tabla 4 sólo la puntuación total del BSQ quedó en la ecuación de regresión, explicando $44 \%$ de la 
Tabla 3. Puntuaciones promedio de los instrumentos y sus factores e indicadores de composición corporal.

\begin{tabular}{lccccccccc}
\hline & \multicolumn{2}{c}{ Muestra total } & \multicolumn{2}{c}{ Riesgo } & \multicolumn{2}{c}{ Sin riesgo } & \multirow{2}{*}{$\mathrm{t}$} & \multirow{2}{*}{$\mathrm{p}$} \\
\cline { 2 - 6 } Bedia & D.E. & Media & D.E. & Media & D.E. & & \\
BSQ & 74.65 & 31.58 & 124.5 & 34.69 & 67.08 & 25.68 & 10.83 & .001 \\
CIMEC & 20.22 & 13.84 & 40.54 & 16.26 & 18.04 & 11.64 & 7.12 & .001 \\
Publicidad & 5.05 & 3.65 & 13.18 & 7.53 & 4.18 & 2.64 & 6.20 & .001 \\
$\begin{array}{l}\text { Malestar con la imagen } \\
\text { corporal }\end{array}$ & 6.02 & 4.70 & 11.89 & 4.18 & 5.34 & 4.30 & 7.80 & .001 \\
Modelo estético & 3.22 & 2.42 & 6.29 & 2.98 & 2.89 & 2.11 & 5.87 & .001 \\
Relaciones sociales & 3.26 & 1.95 & 4.18 & 2.18 & 1.91 & 3.16 & 2.63 & .01 \\
Índice de masa corporal & 22.90 & 4.13 & 24.21 & 3.95 & 22.75 & 4.12 & 1.78 & .07 \\
Porcentaje de grasa & 27.99 & 8.19 & 31.62 & 7.88 & 27.59 & 8.14 & 24.9 & .01 \\
\hline
\end{tabular}

Nota. $B S Q=$ Cuestionario de Imagen Corporal; $C I M E C=$ Cuestionario de Influencias del Modelo Estético Corporal

Tabla 4. Análisis de regresión para riesgo de $A N o B N$.

\begin{tabular}{lcccc}
\hline Variables & $\beta$ & $t$ & $R^{2}$ & $F$ \\
\hline Variable criterio: riesgo de AN & & & & \\
Insatisfacción corporal & .67 & $3.08^{* *}$ & .44 & $222.65^{* *}$ \\
& & & & \\
Variable criterio: riesgo de BN & & & & \\
Insatisfacción corporal & .56 & $7.39^{* *}$ & & \\
Influencia de la publicidad & .15 & $2.05^{*}$ & .42 & $67.25^{* *}$ \\
Índice de masa corporal & .09 & $1.98^{*}$ & & \\
\hline
\end{tabular}

Nota. $* \mathrm{p}<.05, * * \mathrm{p}<0.001$

variabilidad de riesgo de AN. En tanto que las variables insatisfacción corporal, influencia de la publicidad e IMC explicaron $42 \%$ de riesgo de $\mathrm{BN}$.

\section{Discusión}

El propósito de la presente investigación fue analizar el papel de la composición corporal, la insatisfacción corporal y el modelo delgadez sobre el riesgo de TCA en mujeres. Considerando que la prevalencia de obesidad es una problemática sanitaria tanto a nivel nacional como internacional, los resultados del análisis de la composición corporal son importantes debido a que se encontró que $24.55 \%$ de las participantes tenían sobrepeso u obesidad. Este resultado es similar al obtenido en otras investigaciones en las que se ha encontrado que entre 18 y $24 \%$ de mujeres jóvenes presentan exceso de peso (Aguilera y Milián, 2006; Franco, Martínez et al., 2010; Mancilla-Díaz et al., 2010; Saucedo-Molina y Unikel, 2010) y se ubica por debajo del porcentaje de mujeres que presentan sobrepeso u obesidad tanto a nivel nacional (71.90\%) como en el estado de Jalisco (73.30\%) de acuerdo con la ENSANUT (Olaiz-Fernández et al., 2006). Sin embargo, debe considerarse que estos porcentajes corresponden a todas las mujeres mayores de 20 años, en tanto que en esta investigación las participantes fueron jóvenes con una edad promedio de 20.51 años. Asimismo, es relevante que aproximadamente $10 \%$ de las participantes presentaron bajo peso, lo cual también supone una condición de riesgo para su salud tanto física como emocional.

En esta investigación se encontró que $9.69 \%$ de las mujeres presentaron riesgo de TCA. Este dato coincide con otros estudios realizados en el estado de Jalisco (Franco, Martínez et al., 2010; Nuño-Gutiérrez et al., 2009) y es inferior comparado con los resultados de investigaciones realizadas en otros estados como Nuevo León (Franco et al., 2007; Unikel et al., 2010) y la Ciudad de México y su zona metropolitana (Mancilla et al., 2007; Unikel et al., 2006). Este hecho puede explicarse debido a que se trata de grandes metrópolis y en el caso de Nuevo León es un estado cercano a Estados Unidos, país en el que cantidades importantes de mujeres presentan conductas alimentarias de riesgo y un culto exacerbado al modelo de delgadez.

En el campo de estudio de los TCA se sabe que una característica de las personas que presentan estas psicopatologías es el miedo excesivo a tener grasa en el cuerpo. Sin embargo, el principal indicador que se ha utilizado para el análisis de la composición corporal es el IMC. Por ello, resultó relevante analizar si la cantidad de grasa corporal se relacionaba con el riesgo de TCA y se encontró que los mayores porcentajes de personas en riesgo se presentaron 
entre las mujeres que tenían elevados o excesivos porcentajes de grasa corporal y entre quienes tenían sobrepeso u obesidad. En otros estudios también se ha encontrado que las personas con sobrepeso u obesidad presentan los mayores porcentajes de conductas alimentarias de riesgo (Saucedo-Molina et al., 2008; Saucedo-Molina y Unikel, 2010; Unikel et al., 2002). Sin embargo, el porcentaje de grasa no fue relevante en la predicción del riesgo de TCA.

El riesgo para desarrollar alguna psicopatología alimentaria es resultado de la interacción de diversos factores y cuantos más factores interactúan mayor es la probabilidad de que se presente el trastorno. En esta investigación se tomaron en cuenta tres factores de riesgo (insatisfacción corporal, influencia del modelo de delgadez y composición corporal). El análisis de regresión mostró que sólo la insatisfacción corporal predijo el riesgo de AN. Mientras que la interacción entre insatisfacción corporal, la influencia de la publicidad y el IMC predijo el riesgo de BN, siendo mayor la aportación de la insatisfacción corporal en ambos modelos. Estos hallazgos coinciden con otras investigaciones en las que la insatisfacción corporal y la influencia de la publicidad se asocian o predicen el riesgo de TCA (Álvarez et al., 1998; Álvarez et al., 2007; Mancilla et al., 2010; Vázquez, Fuentes, Báez, Álvarez y Mancilla, 2002; Vázquez et al., 2005; Unikel, Aguilar y Gómez-Peresmitré, 2005). En esta investigación se encontró que el IMC por sí sólo no predice el riesgo de TCA, tal como se informa en otros estudios (Byely, Archibald, Graber y Brooks-Gunn, 2000; Cooley y Toray, 2001); sin embargo, al interactuar con la insatisfacción corporal y la influencia del modelo de delgadez su contribución fue relevante. Considerando estos hallazgos puede suponerse que las personas que se encuentran alejadas del ideal corporal prevalente en nuestra sociedad (e.g. sobrepeso u obesidad) pueden ser más vulnerables a la influencia de la publicidad que directa o indirectamente promueve el modelo de delgadez, por tanto presentan insatisfacción corporal, y esta condición las ubica en riesgo de desarrollar alguna psicopatología alimentaria.

En la actualidad puede identificarse una situación paradójica relacionada con el peso corporal. Por un lado, tenemos el aumento en la prevalencia del sobrepeso y la obesidad; y por otro lado, el refinamiento del modelo estético que valora la delgadez como el ideal de belleza, el cual ha permeado en todos los sectores de la población. Sin duda, en la búsqueda de este ideal una cantidad importante de mujeres se sienten inconformes con las dimensiones de su cuerpo, tal como lo demuestran los resultados de esta investigación en la que se identificó que $12.10 \%$ de las mujeres estaban insatisfechas con su cuerpo. Asimismo, se encontró que la insatisfacción corporal fue el factor de riesgo común tanto para AN como para BN. Este resultado apoya la idea de que la insatisfacción con el peso corporal funciona como uno de los principales factores de riesgo de los TCA (Stice, 2002; Toro, 2004).

Considerando los hallazgos de la presente investigación puede concluirse que la insatisfacción corporal mostró ser una variable relevante para el riesgo tanto de $\mathrm{AN}$ como de $\mathrm{BN}$, y que la influencia de la publicidad y del IMC también fue importante para el riesgo de BN. Esta información puede ser considerada para la elaboración e implementación de programas preventivos. No obstante, es necesario llevar a cabo investigaciones que contribuyan a identificar qué otras variables están relacionadas con el riesgo de TCA, lo cual permitirá proponer estrategias de prevención más efectivas tanto a nivel estatal como nacional.

\section{Referencias}

Aguilera, M. A. y Milián, F. (2006). Mapa nutricio y riesgo de trastornos de la conducta alimentaria en adolescentes de la Ciudad de Querétaro, Qro., México. Revista Salud Pública y Nutrición, 7, 1-9.

Álvarez, D. T., Franco, K., Mancilla, J. M., Álvarez, G., y López, X. (1998). Factores predictores de la sintomatología de los trastornos alimentarios. Revista Psicología Contemporánea, 7, 26-35.

Álvarez, G., Franco, K., Vázquez, R., Escarria, A. R., Haro, M., y Mancilla, J. M. (2003). Sintomatología de trastorno alimentario: comparación entre hombres y mujeres estudiantes. Psicología y Salud, 13, 245-255.

Álvarez, G., Mancilla, J. M., Vázquez, R., Unikel, C., Caballero, A., y Corona, D. (2004). Validity of the Eating Attitudes Test: A study of mexican patients with eting disorders. Eating and Weight Disorders, 9, 243-248.

Álvarez, G., Nieto, M. L., Mancilla, J. M., Vázquez, R., y Ocampo, M. T. (2007). Interiorización del ideal de delgadez y sintomatología de trastorno alimentario en mujeres adultas. Psicología y Salud, 17, 251-260.

Álvarez, G., Vázquez, R., Mancilla, J. M., y Gómez-Peresmitre, G. (2002). Evaluación de las propiedades psicométricas del Test de Actitudes Alimentarias (EAT-40) en mujeres mexicanas. Revista Mexicana de Psicología, 19, 47-56.

Barriguete-Meléndez, J. A., Unikel-Santoncini, C., Aguilar-Salinas, C., Córdova-Villalobos, J. A., Shamah, T. y Barquera, S. (2009). Prevalence of abnormal eating behaviors in adolescents in Mexico (Mexican National Health and Nutrition Survey 2006). Salud Pública de México, $51,638-644$.

Bojórquez, I., y Unikel, C. (2004). Presence of disordered eating among Mexican teenage women from semi-urban area: Its relation to the cultural hypothesis. European Eating Disorders Review, 12, 197-202.

Byely, L., Archibald, A. B., Graber, J., y Brooks-Gunn, J. (2000). A prospective study of familial and social influences on girls' body image and dieting. International Journal of Eating Disorders, 28, 155-164.

Cattarin, J. A., y Thompson, J. K. (1994). A 3-year longitudinal study of body image, eating disturbance, and general psychological functioning in adolescent females. Eating Disorders, 2, 114-125.

Chávez, A., Macías, L., Gutiérrez, R., Martínez, C., y Ojeda, D. (2004). Trastornos alimentarios en jóvenes guanajuatenses. Acta Universitaria, $14,17-24$

Cooley, E. y Toray, T. (2001). Disordered eating in college freshman women: A prospective study. Journal of American College Health, 49, 229-235. 
Cooper, P. J., Taylor, M. J., Cooper, Z., y Fairburn, C. G. (1987). The development and validation of the body shape questionnaire. International Journal of Eating Disorders, 6, 485-494.

Franco, K., González, O., Díaz, F., López-Espinoza, A., Martínez, A. G. y Aguilera, V. G. (2010). Reliability and validity of Bulimic Investigatory Test Edinburgh on Mexican women. Journal of Behavior, Health y Social Issues, 2, 25-28.

Franco, K., Luis, P., Mancilla, J. M., Álvarez, G., Vázquez, R., y López, X. (2007). Sintomatología de trastornos del comportamiento alimentario en universitarias de cinco estado de la República Mexicana. Revista Mexicana de Psicología, Número especial, 162-163.

Franco, K., Martínez, A. G., Díaz, F. J., López-Espinoza, A., Aguilera, C., y Valdés, E. (2010). Conductas de riesgo y sintomatología de trastorno del comportamiento alimentario en estudiantes universitarios del Sur de Jalisco, México. Revista Mexicana de Trastornos Alimentarios, 1, 100-108.

Garner, D., y Garfinkel, P. (1979). The Eatting Attitudes Test: An index of the symptoms of anorexia nervosa. Psychological Medicine, 9, 273-279.

Gruber, A. J., Pope, H. G., Lalonde, J. K. y Hudson, J. I. (2001). Why do young women diet? The roles of body fat, body perception, and body ideal. Journal of Clinical Psychiatry, 62, 609-611.

Henderson, M., y Freeman, C. P. (1987). A self-rating scale for bulimia: The BITE. British Journal of Psychiatry, 150, 18-24.

Mancilla-Díaz, J. M., Franco-Paredes, K., Vázquez-Arévalo, R., LópezAguilar, X., Álvarez-Rayón, G. L., y Téllez-Girón, M. T. (2007). A two-stage epidemiological study on prevalence of eating disorders in female university students in Mexico. European Eating Disorders Review, 15, 463-470.

Mancilla-Díaz, J. M., Lameiras-Fernández, M., Vázquez-Arévalo, R., Álvarez-Rayón, G., Franco-Paredes, K., López-Aguilar, X. (2010). Influencias socioculturales y conductas alimentarias no saludables en hombres y mujeres de España y México. Revista Mexicana de Trastornos Alimentarios, 1, 36-47.

Mancilla-Díaz, J. M., López-Aguilar, X., Franco-Paredes, K., ÁlvarezRayón, G., Vázquez-Arévalo, R., y Ocampo, M. T. (2009). Effect of gender and school level on disordered eating behaviours and attitudes in Mexican adolescents. Eating and Weight Disorders, 14, 169-175.

Martínez, S. B., Alvarez, G., Góngora, J., Franco, K., y Mancilla, J. M. (2009). Comparación entre adolescentes con riesgo de trastorno del comportamiento alimentario. Revista Mexicana de Psicología, Número especial, 1, 687-688.

Nuño-Gutiérrez, B. L., Celis-de la Rosa, A., y Unikel-Santoncini, C. (2009). Prevalencia y factores asociados a las conductas alimentarias de riesgo en adolescentes escolares de Guadalajara según sexo. Revista de Investigación Clínica, 61, 286-293.

Olaíz-Fernández, G., Rivera-Dommarco, J., Shamah-Levy, T., Rojas, R., Villalpando-Hernández, S., Hernández-Ávila, M.(2006). Encuesta Nacional de Salud y Nutrición 2006. Cuernavaca, México: Instituto Nacional de Salud Pública.

Organización Mundial de la Salud. Régimen alimentario, nutrición y actividad física. $132^{a}$. Sesión del Comité Ejecutivo, Washington, D. C. 23-27 de junio de 2003.

Pérez, J. A., Valdez, M., y Serrano, S. (2006). Encuesta Nacional de Juventud 2005. Resultados preliminares. Centro de Estudios en Investigaciones sobre juventud. México: Instituto Mexicano de la Juventud.
Saucedo-Molina, T. J., y Gómez, G. (2004). Modelo predictivo de dieta restringida en púberes mexicanas. Rev Psiquiatria Fac Med Barna, 31, 69-74.

Saucedo-Molina, T. J., y Unikel, C. (2010). Conductas alimentarias de riesgo, interiorización del ideal estético de delgadez e índice de masa corporal en estudiantes hidalguenses de preparatoria y licenciatura de una institución privada. Salud Mental, 33, 11-19.

Saucedo-Molina, T. J., Escamilla-Talón, T. A., Portillo-Noriega, I. E., PeñaIrecta, A., y Calderón-Ramos, Z. (2008). Distribución e interrelación de factores de riesgo asociados a trastornos de la conducta alimentaria en púberes hidalguenses, hombres y mujeres, de 11 a 15 años de edad. Revista de Invesigación Clínica, 60, 231-240.

Stice, E. (2002). Risk and maintenance factors for eating pathology: A meta-analytic review. Psychological Bulletin, 128, 825-848.

Toro, J. (2004). Riesgos y causas de la Anorexia Nerviosa. España: Ariel

Toro, J., Salamero, M., y Martínez, E. (1994). Assessment of sociocultural influence on the aesthetic body shape model in anorexia nervosa. Acta Psiquiátrica Scandinavica, 89, 147-151.

Unikel, C., Aguilar, J., y Gómez-Peresmitré, G. (2005). Predictors of eating behaviors in a sample of Mexican women. Eating and Weight Disorders, 10, 33-39.

Unikel, C., Saucedo-Molina, T., Villatoro, J., y Fleiz, C. (2002). Conductas alimentarias de riesgo y distribución del índice de masa corporal en estudiantes de 13 a 18 años. Salud Mental, 25, 49-57.

Unikel, C., Villatoro, J. A., Medina-Mora, M. E., Fleiz, C., Alcántar, E. N., y Hernández, S. A. (2000). Conductas alimentarias de riesgo en adolescentes mexicanos. Datos en poblacón estudiantil del distrito federal. Revista de Investigación Clínica, 52, 140-147.

Unikel-Santoncini, C., Bojórquez-Chapela, I., Villatoro-Velázquez, J., Fleiz-Bautista, C., y Medina-Mora, M. E. (2006). Conductas alimentarias de riesgo en población estudiantil del Distrito Federal: tendencias 1997-2003. Revista de Investigación Clínica, 58, 15-27.

Unikel-Santoncini, C., Nuño-Gutiérrez, B., Celis-de la Rosa, A., SaucedoMolina, T. J., Trujillo, E. M., y García-Castro, F. (2010). Conductas alimentarias de riesgo: prevalencia en estudiantes mexicanas de 15 a 19 años. Revista de Investigación Clínica, 62, 424-432.

Vázquez, R., Álvarez, G., y Mancilla, J. M. (2000). Consistencia interna y estructura factorial del cuestionario de influencia de los modelos estéticos corporales (CIMEC), en población mexicana. Salud Mental, 23, 18-24.

Vázquez, R., Fuentes, E. S., Báez, M. V., Alvarez, G. L., y Mancilla, J. M. (2002). Influencia de los modelos estéticos corporales en la presencia de sintomatología de trastorno alimentario. Psicología y Salud, 12, 73-82.

Vázquez, R., Galán, J., Alvarez, G., Mancilla, J. M., Franco, K., y López, X. (2004). Validez del Body Shape Questionnaire en mujeres mexicanas. Revista Mexicana de Psicología, Número especial, 1, 152.

Vázquez, R., López, X., Álvarez, G., Franco, K., y Mancilla, J. M. (2004). Presencia de trastornos de la conducta alimentaria en hombres y mujeres mexicanos: algunos factores asociados. Psicología conductual, 12, 415-427.

Vázquez, R., Mancilla, J. M., Mateo, C., López, X., Álvarez, G. L. y Ruiz, A. O. (2005). Trastornos del comportamiento alimentario y factores de riesgo en una muestra incidental de jóvenes mexicanos. Revista Mexicana de Psicología, 22, 53-63. 
